\title{
El uso de personajes famosos en las campañas de screening de cáncer de colon
}

Jose A. Martínez I josean.martinez@upct.es

Universidad Politécnica de Cartagena

Manuel Ruiz | manuel.ruiz@upct.es

Universidad Politécnica de Cartagena

María del Carmen Vivo I mariac.vivo@carm.es

Consejería de Sanidad y Política Social de la Región de Murcia.

Resumen: En esta investigación estudiamos la efectividad de la campaña de comunicación sobre la concienciación y prevención del cáncer de colon en España, y los personajes famosos utilizados, con el fin de analizar la idoneidad de su elección. En un experimento con una población mayor de 50 años, encontramos que la imagen de los personajes famosos utilizados no es adecuada. Además el grado de concienciación de la enfermedad no varía ante diferentes estímulos publicitarios. Los resultados de esta investigación deben ayudar a plantear una campaña de comunicación que incremente la tasa de respuesta en el cribado del cáncer de colon.

Palabras clave: psicología social, marketing de la salud, cáncer colorrectal, campañas de prevención, experimento.

Abstract: In this research we analyze the effectiveness of the mass media communication campaign on awareness and prevention of colorectal cancer in Spain, also we analyze use and adequacy of celebrities in this campaigns. In an experiment with a population over 50 years, we find that image of celebrities is not such as good as expected. Furthermore, the degree of awareness of the disease don't change to the different advertising. Results from this research, must help us to carry out mass media campaigns in order to increase the response rate in screening for colon cancer.

Key words: social psychology; health marketing; colorectal cancer; prevention campaigns, experiment. 


\section{Introducción}

El cáncer de colon es uno de los cánceres con mayor incidencia en España. Es la segunda causa de muerte por cáncer, después del de pulmón en el hombre, y del de mama en la mujer. La tasa de supervivencia puede llegar al $90 \%$ en aquellas personas que han tenido una detección temprana del cáncer, siendo la media actual en España en torno al 50\% a los 5 años del diagnóstico. Actualmente, existen diversas formas de detección de esta enfermedad: test de sangre oculta en heces, análisis de sangre y orina, tacto rectal, colonoscopia y biopsia, pero muy pocos cánceres de colon son detectados en un estadio temprano, fundamentalmente porque muchos individuos en edad de riesgo no se realizan la prueba de cribado. Los motivos de la no realización de esa prueba son diversos (Stacey, Torrence y Mitchell, 2008: 239): desconocimiento, olvido, miedo... El hecho de que este cáncer tenga una tasa muy baja de detección precoz hace que se trabaje en campañas de comunicación que permitan crear en la población diana (mayores de 50 años) una mayor concienciación sobre el riesgo de padecer la enfermedad y las bondades de las medidas preventivas existentes.

Sin embargo, los programas piloto de detección precoz implementados en diversas zonas geográficas de España obtienen ratios de aceptación entre el 26 y 47,7\% (Borrás et al, 2010: 11), lo que es insuficiente para llegar al umbral necesario que permita implantar con solidez programas de detección a nivel general, que algunas fuentes sanitarias sitúan en el $60 \%$. Aunque superar el $40 \%$ de aceptación es realmente meritorio, no se llegan a los niveles de otros países como Dinamarca, Inglaterra, Francia o Suecia (Nielson y Whines, 1995:365), Holanda (Hol., 2010:64), Italia (www.osservatorionazionalescreening.it), o Estados Unidos (M. Potter, comunicación personal, 3 febrero, 2011). Por tanto, ni los esfuerzos realizados por parte de los servicios sanitarios públicos en su ámbito geográfico de incidencia ni las campañas de comunicación difundidas por diversas organizaciones a nivel general, han producido hasta el momento el efecto esperado en la población de riesgo. Bien es cierto que existe gran variabilidad en el éxito de esos programas en España, donde se ha propuesto diversas fórmulas de contacto directo con la población diana con resultados dispares (Courtier et al., 2002:209).

La campaña de comunicación de masas realizada en España está promovida por la Alianza para la Prevención del Cáncer de Colon (APCC), una organización formada por varias instituciones relacionadas con la salud. Desde 2009, esta organización promueve una campaña usando diversos medios de comunicación, como televisión, radio o publicidad exterior (www.alianzaprevencioncolon.es).

El uso de esos personajes famosos en esas campañas se justifica por los efectos psicológicos que producen sobre el público objetivo. En este caso, las autoridades sanitarias y las entidades afiliadas que promueven las campañas pretenden incrementar la concienciación sobre la enfermedad, y persuadir a la población diana de acometer el comportamiento socialmente deseado (realizar el screening). Esos objetivos son más probables de conseguir 
usando celebridades que apadrinen y promuevan el mensaje. Por ejemplo, cuando la estrella de baloncesto Magic Johnson anunció en 1991 que era portador el virus del SIDA, se incrementaron los niveles de concienciación de la enfermedad en Estados Unidos y el riesgo percibido de manera importante, especialmente entre la gente joven (Basil y Brown, 2004: 167). Además, muchos individuos cambiaron su comportamiento al ser más precavidos en las situaciones de riesgo de contagio de SIDA. Y es que como Agrawal y Kamakura (1995:56) sostienen, el uso de las celebridades en comunicación incrementa la credibilidad de los mensajes, aumenta el recuerdo y el reconocimiento de las marcas anunciadas, mejora la actitud hacia la organización que vende el producto, e incluso incrementa la probabilidad de compra. En el caso de mensajes relacionados con el cuidado de la salud, como es la prevención del cáncer de colon, el mensaje se convierte en el producto ofrecido, buscando su aceptación por parte del público al que va dirigido.

Sin embargo, la elección de los personajes famosos que actúan como padrinos es importante, porque la efectividad de ese apadrinamiento depende en parte de la identificación del personaje con el público objetivo. Por tanto, y de manera general, las celebridades elegidas deben ser admiradas y respetadas por la audiencia, y tener un estatus mayor que ésta (Basil y Brown, 2004: 161). Además, no siempre tienen un efecto beneficioso sobre el producto que apadrinan. Por ejemplo, ciertas conductas de esos personajes pueden dañar a la marca, hasta el punto de que ésta rompa su relación con ellos. Casos como el del golfista Tiger Woods y su ruptura con Gillete, Accendture, AT\&T Gatorade o el del jugador de baloncesto Latrell Sprewell, que trató de asfixiar a su entrenador su posterior ruptura con Converse (Ruihley, Runyan y Lear, 2010: 135), son muy ilustrativos. En otras ocasiones, una celebridad anuncia tantos productos que se produce una pérdida de credibilidad, sobre todo en aquellas apariciones para apadrinar productos que tienen menos relación con la actividad principal del famoso, o la audiencia puede tener la impresción de que está realizando ese anuncio únicamente por recibir una sustancial cantidad económica, lo que puede traducirse en un efecto de vampirización, donde se recuerda el famoso pero no el producto (Dohl, Molchanov y Stork, 2011:157).

La población diana requiere conocer la enfermedad y los métodos de prevención, es decir, necesita tener mucha información al respecto para acceder a realizarse la prueba de cribado (Jepson, Hewison, Thompson y Weller, 2005:193). Sin embargo, en ocasiones, el adecuado conocimiento no es suficiente para que la población de riesgo realice el screening (Weinberg et al., 2009:227); se necesita que los mensajes sean lo más persuasivos posibles. Y esta es precisamente una de las razones del uso de celebridades en este tipo de campañas de comunicación.

Por tanto, el primer paso para crear una campaña de comunicación exitosa es incrementar la concienciación de la enfermedad. Realizar una campaña de comunicación de masas (utilizando además celebridades) es una manera recomendable de conseguir ese objetivo. Los mecanismos psicológicos de la mera exposición y de la fluencia explican por qué los mensajes altamente repetidos y las personas percibidas como familiares (celebridades) son 
preferidos por los individuos (Pandelaere, Millet y Van den Bergh, 2010:2; Pocheptova, Labroo y Dhar, 2010:29). Concienciación y persuasión deben actuar de manera paralela, con el fin de que incrementar la probabilidad de acometer el comportamiento socialmente deseable.

El uso de famosos en las campañas de prevención del cáncer de colon es un denominador común en muchos países. Por ejemplo, en Estados Unidos, la campaña "Screen For Life" se lanzó 1999 para concienciar sobre la enfermedad y los beneficios de la detección precoz (Cooper, Gelb, Jameson, Macario, Jorgensen y Seef, 2005:386). Diversos actores famosos, como Jimmy Smits o Morgan Freeman, entre otros, han apadrinado esta campaña desde entonces. En Alemania, desde 2001, la Fundación Félix Burda trata de concienciar a la población sobre la necesidad de la prevención. Tienen apoyos de distintas celebridades, políticos y científicos. Organiza unos premios anuales que son presentados por celebridades y realizan campañas de comunicación masiva, donde han utilizado a personajes famosos: deportistas (Michael Schumacher), cómicos (Atze Schröder) o actrices (Annette Frier), entre otros. En 2003 se hizo el primer programa de televisión dedicado a la prevención de cáncer de colon, donde acudieron famosos alemanes y se presentaron testimonios en defensa de la prevención de esta enfermedad. Además, un famoso se hace la prueba en directo, evento que está basado en el show que organizó la conocida presentadora estadounidense Katie Courcic en 2000 (Schroy, Glick, Ronbinson, Lydotes, Evans y Emmons, 2008:7). En Inglaterra, la organización NHS para la prevención del cáncer ha utilizado en sus campañas a la mujer del futbolista Bobby Moore, fallecido por esta enfermedad. En Italia, el actor Renato Pozzeto y en Australia el deportista Max Walker, también han apadrinado campañas de concienciación y persuasión.

En España, en 2009, la APCC lanzó la primera campaña nacional de concienciación y persuasión, utilizando a la escritora Carmen Posadas y al torero José Ortega Cano como prescriptores. La agencia de publicidad NCA y Asociados ganó el concurso público para realizar la campaña. Tras considerar varias opciones creativas, la agencia se decantó por utilizar prescriptores famosos. Primeramente se pensó en utilizar periodistas de renombre para incrementar la credibilidad del mensaje, incluso se hizo una primera lista de 20 hombres y 20 mujeres. Sin embargo, la Asociación Española Contra el Cáncer (AECC) tenía ya acuerdos con diversos personajes, optándose finalmente por esas dos celebridades, que a su vez, representaban dos estratos sociales diferentes. Tanto Ortega Cano como Posadas no cobraron nada por su colaboración, accediendo amablemente a ayudar prestando su imagen en diferentes anuncios publicitarios.

Sin embargo, los esfuerzos en la elaboración de las campañas de comunicación no se corresponden con la evaluación de su eficacia. En España, sólo se obtuvo información acerca de los impactos de audiencia a través del panel de audímetros de Sofres, pero esos datos no dicen nada acerca de la eficacia en cuanto a notoriedad (recuerdo espontáneo y sugerido), ni en cuanto a la actitud hacia los prescriptores. Ésta es una práctica habitual en otros países. Sólo en Estados Unidos, al margen de la utilización de los impactos de audiencia, existen 
investigaciones que tratan de evaluar la eficacia de las campañas de concienciación, como el estudio realizado tras la primera colonoscopia de Katie Courcic (Cram, Fendrick, Inadomi, Cowen, Carpenter y Vijan, 2003:1602). La única investigación en España que va más allá de los datos sobre audiencia es la promovida por la AECC a través de su Observatorio, e implementada por la consultora Advira. Esa investigación, realizada en otoño de 2010 estudia el nivel de concienciación de la enfermedad, concluyendo que es insuficiente, ya que otros tipos de cáncer son percibidos como de mayor prevalencia y gravedad (pulmón, mama y próstata). No obstante, como comentan Jorgensen, Gelb, Merrit y Seef (2001:421), la carencia de recursos es una barrera para profundizar en las medidas de eficacia de las campañas del cáncer de colon.

El objetivo de esta investigación es evaluar la imagen de los famosos utilizados en la campaña de comunicación en España, con el fin analizar la idoneidad de su elección. Para ello, en primer lugar, partimos de la hipótesis de que la asociación que la población diana pueda hacer sobre Carmen Posadas y el cáncer es débil, ya que no es un personaje extremadamente popular y la vinculación a su marido (Mariano Rubio, antiguo gobernador del Banco de España que murió de cáncer de colon en 1999) poco conocida para el gran público. En segundo lugar, y aunque la relación con el càncer de Ortega Cano es más evidente (su mujer, la cantante Rocío Jurado, murió de cáncer de páncreas en 2006), dado la imagen de Ortega Cano se ha visto afectada negativamente en los últimos años por diversos asuntos relacionados con la prensa rosa, establecemos la hiópótesis de que la credibilidad y la confianza que pueda generar el torero en la población pueda estar en entredicho. Asimismo, valoramos el grado de notoriedad de la enfermedad, utilizando el recuerdo espontáneo y sugerido (Kholi, Luethesser y Suri, 2007:416), y su vinculación con la aparición de los anuncios donde aparecen estos personajes famosos.

Por tanto, esta investigación contribuye de forma novedosa a la literatura sobre comunicación publicitaria y psicología de la salud en el ámbito del cáncer de colon, de la manera siguiente: (1) Estudia la imagen de los personajes famosos utilizados para apadrinar una campaña de concienciación y persuasión; (2) Analiza el grado de concienciación sobre la enfermedad y su relación con los estímulos publicitarios. Así, los resultados de esta investigación deben ayudar a plantear la idoneidad de la campaña de comunicación, con el fin de optimizar todas las herramientas de las que disponen las entidades públicas para tratar de incrementar la tasa de respuesta en la prueba de cribado del cáncer de colon. Como Yanovitzky y Blitz (2000:118) afirman, incluso pequeñas mejoras en los índices de participación de este tipo de pruebas son muy importantes.

\section{Método}

\subsection{Muestra}

Se recogieron datos de encuestas personales realizadas en abril de 2010 a un total de 183 personas pertenecientes a la población diana, con una edad media de 64 años, de los cuales 
un $\mathbf{7 3 . 6 \%}$ eran mujeres y un $26.4 \%$ eran hombres. Todos los participantes eran alumnos de la Universidad de Mayores, cursos dirigidos a ese segmento de edad organizados por la Universidad Politécnica de Cartagena. Se eligió esta población debido a la estrategia de expansión de los estudios de cribado que realiza la Consejería de Sanidad de la Región de Murcia. Como indican Pérez-Riquelme et al. (2008:17), diversas localizaciones geográficas de la Región de Murcia se incorporan secuencialmente a las pruebas piloto. La comarca de Cartagena era una de las próximas a ser incluidas en esa futura expansión al momento de inicio de este estudio.

\subsection{Instrumentos}

La eficacia de la publicidad se midió a través de 3 variables; recuerdo espontáneo, recuerdo sugerido e imagen del personaje que protagonizaba el anuncio. Tratamos de analizar cómo los participantes valoraban el riesgo percibido de padecer cáncer de colon en relación a otros tipos de cáncer. Para ello, les pedimos que indicaran ordenadamente los tres tipos de cáncer que más riesgo creían que podían padecer, en un procedimiento parcialmente basado en el utilizado por McGarrity, Long, Peiffer, Converse y Kreig (1989:142). A un grupo de participantes (primero y tercero) no les dábamos ninguna relación de tipos de cáncer, mientras que al otro grupo (segundo y cuarto) sí. La relación de cánceres elegidos fue realizada atendiendo a los más frecuentes para hombres y mujeres en la Región de Murcia (Pérez-Riquelme, et al., 2008:14): colon-recto, estómago, laringe, linfoma, mama, próstata, pulmón, vejiga, útero, dejando la opción abierta de "otros", para que los encuestados libremente pudieran nombrar otro tipo de cáncer que no aparecía en el listado. De este modo, se obtenía información no sólo sobre la eficacia de la publicidad, sino sobre el nivel de concienciación.

En relación a la evaluación de la imagen, se les pidió a los participantes que valoraran a los protagonistas de la campaña publicitara: Carmen Posadas y Ortega Cano. Así se les realizó tres preguntas referentes a su nivel de agrado, confianza y credibilidad, que son tres variables fundamentales para analizar el atractivo del personaje famoso (Von Hippel y Trivers, 2011:5), y por ende, su imagen proyectada. Recordemos que a mayor nivel de atracción, mayor es la probabilidad de identificación del sujeto con el protagonista del anuncio, y por tanto, mayor es la probabilidad de que el mensaje sea efectivo. Se utilizaron escalas diferencial semántico de siete opciones de respuesta.

Una de las cuestiones planteadas al inicio de la investigación fue la idoneidad de la elección de los personajes. Dadas las razones por las que éstos han sido protagonistas, uno de los objetivos fue valorar si efectivamente esos personajes cumplían con la misión por la que fueron elegidos. Por ello, preguntamos sobre el conocimiento de los participantes sobre la actividad profesional de Carmen Posadas y acerca de la identidad de su marido. Asimismo, y dado que la imagen de Ortega Cano se ha visto salpicada de muchos problemas, queríamos comparar ésta con la de otros personajes famosos, en su misma banda de edad, y que podían también ser susceptibles de ser utilizados para esta campaña. Esos personajes 
fueron: Arturo Pérez Reverte, Matías Prats, Johan Cruyff, Imanol Arias y Plácido Domingo. La elección de estos personajes fue realizada atendiendo a los siguientes criterios: Pérez Reverte es natural de Cartagena (al igual que Ortega Cano), ciudad de los encuestados, Matías Prats ha protagonizado numerosas campañas de publicidad, Johan Cruyff ha realizado campañas relacionadas con el cuidado de la salud, Imanol Arias ha realizado campañas promovidas por organizaciones no gubernamentales, y Plácido Domingo ha padecido recientemente cáncer de colon. Además, tanto Imanol Arias como Matías Prats fueron primeramente propuestos por los creativos de NCA y Asociados como candidatos a apadrinar la campaña, fundamentalmente por la credibilidad que transmiten.

El diseño experimental fue realizado de la siguiente forma. Se propusieron cuatro tratamientos diferentes. En los tratamientos 1 y 3 se les preguntaba por el recuerdo espontáneo, con la diferencia de que en el tratamiento 3 esa pregunta era realizada después de que los participantes hubieran visto y valorado a los personajes (por tanto podría existir un efecto de la publicidad sobre el nivel de recuerdo). En los tratamientos 2 y 4 se les preguntaba por el recuerdo sugerido, con la misma salvedad anterior para el tratamiento 4. Es importante resaltar que para evitar un posible efecto de dependencia en las respuestas sobre la imagen de los personajes, éstas fueron aleatorizadas en su orden en cada cuestionario, por lo que se controló ese posible sesgo. Los personajes eran mostrados sin hacer alusión al mensaje de la campaña, para no condicionar las respuestas de recuerdo espontáneo y sugerido. No obstante, se respetaba el diseño original de los anuncios de publicidad gráfica. La inclusión del resto de personajes se hizo realizando fotomontajes para que su aparición tuviera exactamente las mismas condiciones gráficas que los protagonistas reales de la campaña (Figura 1 ).

Finalmente, se les preguntaba a los encuestados si tenían familiares o personas queridas con la enfermedad, dado que esto incrementaría la familiaridad con el cáncer de colon, y podría condicionar las respuestas.

\section{Resultados}

Primeramente, analizamos las percepciones de riesgo de padecer la enfermedad en base a la condición de recuerdo espontáneo (tratamientos 1 y 3). Para ello, creamos un índice mediante las puntuaciones asignadas a la percepción del riesgo de padecer la enfermedad de los encuestados. Así, asignamos 3 puntos a la enfermedad que era percibida por los encuestados como la de más riesgo de padecer, con 2 puntos a la percibida en segundo lugar, 1 punto a la percibida en tercer lugar, y 0 puntos si ese tipo de cáncer no era nombrado.

Como se observa en la Tabla 1, tras aplicar la prueba de Mann-Whitney, no existen diferencias significativas $(p=.67)$ en la percepción de riesgo que los participantes tienen sobre la enfermedad entre los tratamientos 1 y 3 . No existen tampoco diferencias para los hombres $(p=.28)$ y las mujeres $(p=.22)$. Y tampoco se encuentran diferencias si se divide la 
muestra en aquellos individuos que tienen antecedentes de la enfermedad $(p=.68)$ frente a los que no los tienen $(p=.56)$.

Posteriormente, analizamos dentro del tratamiento 1 si existían diferencias en la valoración del riesgo de las diferentes tipos de cáncer. Para ello, sumamos los valores de ordenación anteriores y los comparamos utilizando la prueba de Wilcoxon para muestras dependientes (Tabla 2). Para los hombres, el riesgo de padecer cáncer de colon está por debajo del de padecer cáncer de próstata $(p=.046)$. Para las mujeres, está por debajo del de mama $(p=.006)$. Las pocas respuestas obtenidas para el resto de cánceres más nombrados hacen que se tenga poca potencia para detectar diferencias significativas, aunque la tendencia es clara: el cáncer de colon tiende a ser percibido como de menor riesgo que el de pulmón en los hombres, y que el de útero, páncreas y pulmón en mujeres. Otros tipos de cánceres como el de piel, hígado, estómago, etc., tuvieron una presencia prácticamente despreciable, y con menor importancia que el de colon. Los resultados obtenidos para el tratamiento 3 son casi idénticos a los obtenidos para el tratamiento 1 (Tabla 2). Por tanto, la presencia de los personajes famosos protagonistas de la campaña no hace cambiar la tendencia en las respuestas frente al tratamiento 1 . Finalmente, uniendo ambos tratamientos se obtiene mayor potencia para los análisis, mostrando como el cáncer de colon es significativamente percibido con menor riesgo que el de pulmón y próstata en los hombres, y que el de pulmón, útero, páncreas y mama en las mujeres (Tabla 2).

Realizamos, a continuación, los mismos análisis pero sobre el recuerdo sugerido. En la Tabla 3 se muestra como, al igual que sucedía con el recuerdo espontáneo, el estímulo de la presencia de los personajes protagonistas de la campaña no cambia significativamente las percepciones de riesgo a nivel general, ni en función del sexo o de la familiaridad con la enfermedad.

Asimismo, y utilizando el mismo procedimiento de asignación numérica realizado anteriormente, en la Tabla 4 se muestran los resultados sobre las diferencias en la valoración de riesgo con respecto a otros tipos de cáncer. En esta ocasión sí que existe una diferencia en relación a los resultados obtenidos con el recuerdo espontáneo, y es que el cáncer de colon se sitúa al mismo nivel de percepción de riesgo que el resto de cánceres de alta prevalencia en la población. Así, no hay diferencias significativas entre los principales tipos de cáncer, ni para los hombres ni para las mujeres.

Finalmente, estudiamos dos de las cuestiones directamente relacionadas con la imagen de los dos personajes seleccionados. En primer lugar, nos planteamos si la notoriedad de Posadas era suficientemente elevada, y si se le vinculaba a su marido (fallecido por cáncer de colon). Para ello, pedimos a los encuestados que indicaran su profesión y el nombre de su marido, respectivamente. El $69 \%$ de los encuestados reconocieron correctamente su profesión ( $62.5 \%$ de los hombres y $72.4 \%$ de mujeres). No obstante, sólo el $39.9 \%$ de los encuestados conocían la identidad de su marido ( $27.0 \%$ de hombres y $45.5 \%$ de mujeres). 
En segundo lugar, analizamos la imagen de los personajes famosos en relación a cinco personajes que se podían haber utilizado como alternativa a Ortega Cano. La Tabla 5 muestra como, efectivamente, el agrado, la confianza y la credibilidad percibida por los encuestados es ostensiblemente menor que el resto de personajes (excepto Johan Cruyff) e incluida Carmen Posadas. Los valores están normalizados en una escala $[0,1]$. Así, se puede construir un índice de imagen global, a partir del promedio de las tres variables anteriores, es decir, construir un nuevo concepto de manera formativa (Edwards y Bagozzi, 2000:157). La Figura 2 muestra la comparación de la imagen global de los personajes. Tras aplicar el test de Wilcoxon, sólo la comparación con Cruyff es no significativa $(p=.23)$, por lo que el resto de personajes tiene mejor imagen que el torero.

\section{Discusión e implicaciones}

En esta investigación hemos analizado la efectividad de la campaña de concienciación y prevención del cáncer de colon en relación a la imagen de los personajes y su efecto sobre la notoriedad de la enfermedad.

En cuanto a la concienciación, los participantes espontáneamente sitúan al cáncer de colon por encima del resto de cánceres, pero por debajo del de próstata y pulmón en hombres y del de útero, páncreas, pulmón y mama en mujeres. Esto indica que el nivel de concienciación en hombres es coherente con la prevalencia de la enfermedad, y coincide con los resultados obtenidos por el Observatorio del Cáncer (2011:4), quienes también miden el recuerdo espontáneo. Sin embargo, para las mujeres, el cáncer de colon no es espontáneamente percibido al nivel de riesgo que debiera. No obstante, la situación cambia ostensiblemente cuando se les sugiere un listado de tipos de cáncer. Entonces sí que el cáncer de colon es situado al mismo nivel de riesgo que el resto, tanto en hombres como en mujeres. Esto indica que una mayor deliberación de los participantes en presencia del nombre "cáncer de colon" activa asociaciones mentales diferentes a las realizadas de manera espontánea, confiriendo a la enfermedad un riesgo bastante alto (incluso al mismo nivel de otros cánceres con más prevalencia). De este modo, sí que los esfuerzos en concienciación realizados en los últimos años por las autoridades sanitarias están teniendo calado en la mente de la población diana, de manera menos superficial ciertamente, pero igualmente valiosa.

Y esa concienciación probablemente está siendo conseguida principalmente por las vías de la comunicación interpersonal con los médicos, y con las acciones de comunicación no directamente relacionadas con la aparición de famosos (como los folletos informativos en las farmacias, por ejemplo). Y es que la estimulación con los personajes famosos de la campaña, no cambia el nivel de recuerdo espontáneo ni sugerido. Esto indica que esos anuncios no han sido notorios ni persuasivos, al menos al nivel que sería deseable. Hay que recordar que la campaña de comunicación de masas tiene su principal actuación en el mes de marzo (mes del cáncer de colon), y que las encuestas fueron realizadas unas pocas semanas después, es 
decir, prácticamente al finalizar la campaña, con lo que los efectos, de existir, no deberían haberse vistos distorsionados por el paso del tiempo.

Probablemente una de las causas de la inexistencia de ese efecto es la cuestionable idoneidad de los dos personajes famosos utilizados. La imagen de Ortega Cano no es la adecuada, al menos en relación a otros personajes que podrían haberse utilizado y que superan ampliamente al torero en cuanto a agrado provocado, y confianza y credibilidad percibida. No ocurre así con Carmen Posadas, que sí que tiene una buena imagen, pero el problema es que hay un porcentaje importante de personas (30\%) que saben que es famosa pero no el porqué (no conocen su profesión), mientras que el $60 \%$ no la vincula a la enfermedad, ya que no la relacionan con su marido.

Por tanto, sería recomendable replantearse las acciones de comunicación masivas, al menos en cuanto a la elección de los padrinos de la campaña. Admitimos, no obstante, sobre esta recomendación que la evaluación de los personajes se ha realizado sin asociarlos a mensajes explícitos sobre el colon. Aunque ello era necesario para no incurrir en un sesgo debido a la posibilidad de condicionar las respuestas de recuerdo espontáneo y sugerido, es cierto que también puede ocasionar otro sesgo relativo a la evaluación de la imagen de los famosos fuera del contexto del anuncio real

Reconocemos que la principal limitación de este estudio reside en el carácter no aleatorio de la muestra considerada. No obstante, los resultados del recuerdo espontáneo son prácticamente idénticos a los obtenidos por el Observatorio del Cáncer (2011:4), quienes utilizan una muestra aleatoria y bastante más numerosa. Esto incrementa la fiabilidad de nuestros análisis. Además, las dificultades para acceder a este segmento de población en este tipo de investigaciones es muy grande, ya que son encuestas que hay que realizar de manera personal, debido a que contienen material gráfico; por tanto, es imposible hacerlas de manera telefónica, como lo ha hecho el Observatorio del Cáncer (2011:4). Y tampoco puede utilizarse los centros de salud, por el condicionamiento de los estímulos recibidos, y porque las encuestas necesitan ser cumplimentadas con la debida calma y sosiego para evaluar bien las preguntas y las imágenes. Obviamente tampoco serían válidas las encuestas postales, por la carencia de control sobre las respuestas. Por tanto, y admitiendo las debilidades de la muestra de conveniencia, nuestro diseño de investigación tiene muchos puntos fuertes. En cualquier caso, aunque los participantes son todos de la Universidad de Mayores, existe heterogeneidad en cuanto a su procedencia social, lo que hace más robustos los resultados obtenidos. No obstante, también admitimos que se necesitaría profundizar más sobre el anàlisis de los mensajes (contenido) y de la repetición de los mismos, en aras de proveer conclusiones más completas sobre la eficacia de la comunicación, algo que próximas investigaciones podrían realizar. 
Finalmente, futuras campañas de comunicación masiva en España deberían también plantearse el uso de personajes anónimos como prescriptores, tal y como sucede en otros países, como Estados Unidos y Alemania, por ejemplo, donde combinan el uso de famosos y no famosos en publicidad en las campañas del cáncer de colon. De este modo, se cubrirían los diferentes niveles de influencia (corto y largo plazo) recientemente propuestos por Zhao y Xie (2011:486), donde los personajes anónimos (similares en edad, profesión o etnia) serían más influyentes en las decisiones a corto plazo que los famosos, más distantes pero más influyentes en decisiones a largo plazo.

\section{Agradecimientos}

Esta investigación ha sido posible gracias a la financiación de la Consejería de Sanidad de la Región de Murcia, y a la colaboración de la Universidad Politécnica de Cartagena. Agradecemos también la ayuda de Michael B. Potter, Kristjan Sigurdsson, Jay Onysko, JeanLuc Bulliard, Verena Schenk-Welker, Grazia Grazzini, Julien Wiggins, Brian Cox e Iris Lansdorp-Vogelaar, por su desinteresada colaboración. Esta investigación está en deuda con Alfredo González de NCA y Asociados, y con Esther Díez de la Asociación Española Contra el Cáncer, por la valiosa información suministrada. Finalmente, mostramos nuestro más profundo respeto a José Ortega Cano y Carmen Posadas, cuya evaluación de la imagen proyectada se ha realizado sin ningún tipo de acritud, y en aras de proveer resultados que mejoren la efectividad de las campañas de prevención del cáncer, sin poner en cuestión en ningún momento, la inestimable valía de su desinteresada colaboración. Extendemos nuestros respetos al resto de personajes famosos utilizados para la evaluación, cuya imagen ha sido utilizada con la imparcialidad que los objetivos científicos justifican.

\section{REFERENCIAS BibLIOGRÁfiCAS}

AgraWAL, Jagdish; KamaKURA, Wagner (1995):"The Economic Worth of Celebrity Endorsers: An Event Study Analysis", en Journal of Marketing, n59, pp(56-62).

BASIL, Michael.D; Brown, William.J. (2004): "Magic Johnson and Mark McGwire: The power of identification with sports celebrities", en Kahle R.; Riley, C , Sports marketing and the psychology of marketing communication, Mahwah: Lawrence Erlbaum Associates Publishers pp 159-171.

Borrás, José. M; CAstells, A; Espinás, José. A; Salas, Dolores ; Pérez-Riquelme, Francisco; Portillo, Isabel; González de ALEdo, Álvaro; REYES, Juana; QuinTERo, Enrique; Segnan, Nereo . (2010): "Prevención del cáncer de colon en España: un reto común". Alianza para la prevención del cáncer de colon. 
Copper, Crystale.P; GelB, Cynthia.A; JAMESOn, Heather; MACARIo, Everly; Jorgensen, Cynthia.M; SeEf, Laura. (2005): "Developing English and Spanish Television Public Service Announcements to Promote Colorectal Cancer Screening" en Health Promotion Practice, n6, pp (385-393).

Coutier, Ricardo; Casamitjana, Montserrat; Maciá, Francesc; Panadés, Agustín; Castells, Xavier; Gil, María José; Hidalgo, José.M; SÁnchez-Ortega, José .M. (2002): "Participation in a colorectal cancer screening programme: Influence of the method of contacting the target population", en European Journal of Cancer Prevention, no11, pp(209-213)

Cram, Peter; Fendrick, A.Mark; Inadomi, John; Cowen, Mark.E; CarPenter, Daniel; VIJAN, Sandeep. (2003): "The impact of a celebrity promotional campaign on the use of colon cancer screening: The Katie Couric effect", en Archives of Internal Medicine, no163, pp(1601-1605).

Ding, Haina; Molchanov, Alexander; Stork, Phillip. A. (2011). "The value of celebrity endorsements: A stock market perspective", en Marketing Letters. 2011, n022, pp(147-163).

EdWARS, Jeffrey.R; BAGOZZI, Richard.P. (2000): "On the Nature and Direction of Relationships Between Constructs and Measures", en Psychological Methods , no 5, $\mathrm{pp}(155-174)$.

Hol, Lieke; Van Leerdam, Monique. E; Van Ballegooijen, Marjolein; Van VuUren, Anek.J; Van DekKen, Harkema; ReIJerink, Jaqueline C.I.Y; Van Der Togt, Alexandra.C; HABBemA, Dik.J.F; KUIPERS, Ernest.J. (2010): "Screening for colorectal cancer: randomised trial comparing guaiac-based and immunochemical faecal occult blood testing and flexible sigmoidoscopy", en An International Journal of Gastroenterology and Hepatology, n059, pp(62-68).

Jepson, Ruth.G; Hewison, Jenny; Thompson, A.G; Weller, D. (2005): "How should we measure informed choice? The case of cancer screening", en Journal of Medical Ethics, n031, $\mathrm{pp}(192-196)$.

Jorgensen, Cynthia.M.; GelB, Cynthia.; Merrit, Tracie.L. y Seef, Laura.C. (2001): "CDC's Screen for Life: A national colorectal cancer action campaign" en Journal of Women's Health \& Gender-Based Medicine, no10, pp(417-423).

KoHLI, Chiranjeev.; LeUthesser, Lance; SuRI, Rajneesh. (2007): "Got slogan? Guidelines for creating effective slogans" en Business Horizons, n05, pp(415422).

Mcgarrity, Thomas.J; Long, Patricia.A; Peeiffer, Laurie.P; Converse Joseph.O; KREIG, Arthur.F. (1989):"Results of a television-advertised public screening 
program for colorectal cancer" en Archives of Internal Medicine, nº149, $\operatorname{pp}(140-144)$.

NeILSON, Aileen.R; WhyNes, David.K. (1995): "Determinants of persistent compliance with screening for colorectal cancer", en Social Science \& Medicine, n041, pp(365-374).

Orbe, Isabel; Fernández, Belen; Fernández, Ana; Cambas, Naiara (2011): "Cáncer Colorrectal Percepciones y barreras ante las pruebas de cribado". Marzo 2011. Disponible:https://www.aecc.es/Investigacion/observatoriodelcancer/Estudios/D ocuments/Informe\%20Preliminar\%20de\%20Resultados\%200ncoBarometro.pdf (Consultado 1/04/2011).

Pandelaere, Mario ; Millet, Kobe; Van Den Bergh, Bram. (2010): "Madonna or Don McLean? THE EFFECT OF ORDER OF EXPOSURE ON RELATIVE LIKING", EN JOURNAL OF CONSUMER PYSCHOLOGY, N02, PP(442-451).

Pérez-Riquelme, Francisco; Cruzado, José; Carballo, luis. F; Torrella, Emilio .A; LÁZARO, María Dolores; Cavas, Mari Carmen. (2008): Prevención del cáncer de colon y recto en la Región de Murcia. Proyecto Piloto. Murcia: Consejería de Sanidad de la Región de Murcia.

PoChePstova, Anastasiya; LABROo, Aparna.A; DhAR, Ravi. (2010): "Making products feel special: When metacognitive difficulty enhances evaluation", en Journal of Marketing Research, n047, pp(1059-1069).

RUIHLEY, Brody J.; RUNYAN, Rodney C.; LEAR, Karen E. (2010). "The use of sport celebrities in advertising: A replication and extension", en Sport Marketing Quarterly, n019, pp(132-142).

SCHRORY, Paul.C; Glick, Julie.T; Robinson, Patricia.A; Lydotes Maria.A; Evans, Stephen.R; Emmons, Karen.E. (2008): "Has de surge in media attention increased public awareness about colorectal cancer and screening?", en Journal of Community Health, n033, pp(1-9).

Stacy, Richard; Torrence, William. A; Mitchell, Carol.R. (2008): "Perceptions of Knowledge, Beliefs, and Barriers to Colorectal Cancer Screening", en Journal of Cancer Education, n023, pp(238-240).

VON HIPPEL, William; TRIVERS, Robert. (2011): "The evolution and psychology of selfdeception", en Behavioral \& Brain Sciences, $n^{\circ} 34, \mathrm{pp}(1-56)$.

Weinberg, David.S; Miller, Suzanne; Rodoletz, Michelle; Egleston, Brian; Fleisher, Linda; Buzaglo, Joanne; Keenan, Eileen; MARKS, Jaime; Bieber, Eric.(2009): "Colorectal Cancer Knowledge is not Associated with Screening Compliance or Intention", en Journal of Cancer Education, no24, pp(225 -232). 
YANOVITZKY, Itzhak; BLITZ, Cynthia.L. (2000): "Effect of media coverage and physician advice on utilization of breast cancer screening by women 40 years and older", en Journal of Health Communications, n5, pp(117-134).

ZHAO, Min; XIE, Jinhong. (2011): "Effects of Social and Temporal Distance on Consumers Responses to Peer Recommendations", en Journal of Marketing Research, no48, pp(486-496).

[Recibido: 18 de noviembre de 2011. Aceptado con cambios: 9 de diciembre de 2012]. Aceptación definitiva: 16 de diciembre de 2012]. 
Figura 1.

Campaña real (no mostrada en la encuesta)

"Sj tienes más de 50 años,
no lo dejes pasar. Habla con tu médieo."
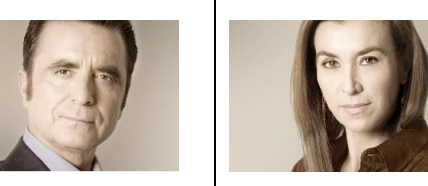

el cáncer de colon se pulede curar en el $90 \%$ de los casos."

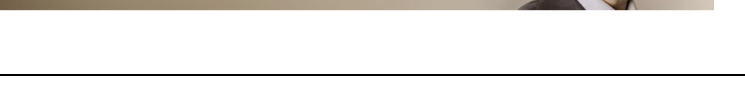

Presentación de los protagonistas de la campaña real en la encuesta

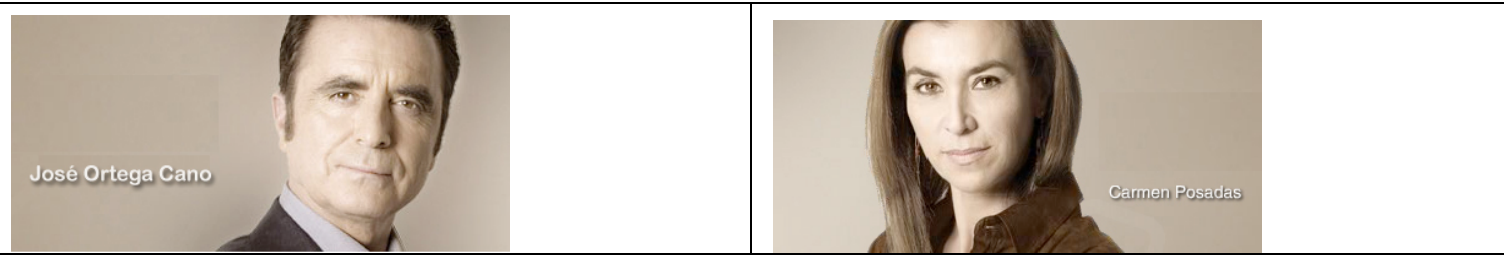

Ejemplo de presentación del resto de personajes famosos en la encuesta

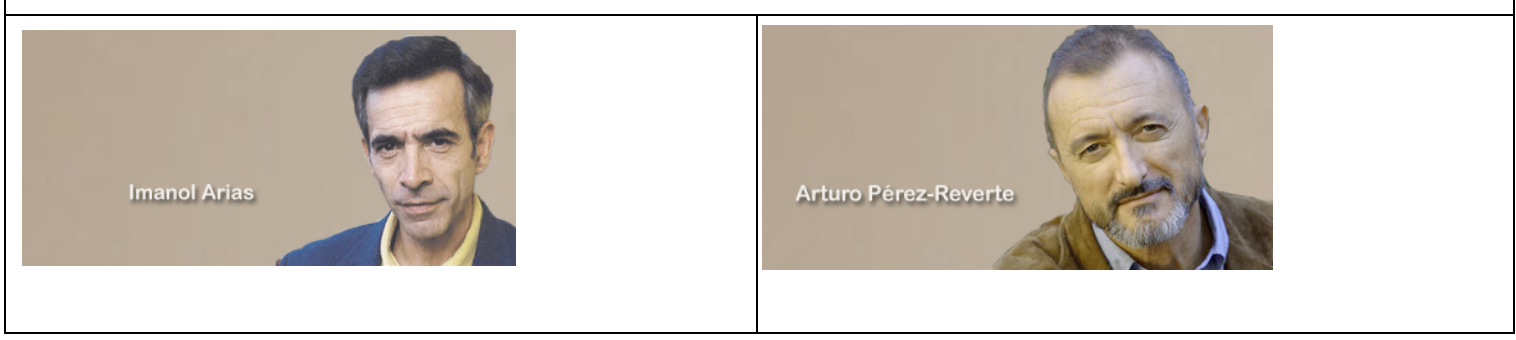

Presentación de los personajes famosos en la encuesta 
Tabla 1

\begin{tabular}{|c|c|c|c|c|c|}
\hline & Total & Hombres & Mujeres & $\begin{array}{l}\text { Sí } \\
\text { antecede }\end{array}$ & $\begin{array}{l}\text { No } \\
\text { antecede }\end{array}$ \\
\hline $\begin{array}{l}\text { Rango promedio } \\
\text { tratamiento } 1\end{array}$ & 37.0 & 10.2 & 26.9 & 18.8 & 17.2 \\
\hline $\begin{array}{c}\text { Rango promedio } \\
\text { tratamiento } 3\end{array}$ & 35.0 & 13.3 & 22.1 & 20.3 & 15.2 \\
\hline$p$ & .67 & .28 & .22 & .68 & .56 \\
\hline
\end{tabular}

Diferencia entre las percepciones de riesgo de padecer cáncer de colon entre los tratamientos 1 y 3. Prueba U de Mann-Whitney. 
Tabla 2

\begin{tabular}{lllllll}
\hline Tratamiento & Hombre & \multicolumn{5}{c}{ Mujeres } \\
& Pulmón & Próstata & Pulmón & Útero & Páncrea & Mama \\
\hline Media & 2.0 & 2.2 & 1.8 & 1.7 & 2.4 & 2.7 \\
Media Colon & .80 & .80 & 1.54 & 1.54 & 1.54 & 1.54 \\
$p$ & .105 & .046 & .120 & .157 & .221 & $.006 * *$ \\
\hline Tratamiento & & & & & & \\
Media & 2.0 & 2.7 & 1.7 & 1.9 & 3.0 & 2.7 \\
Media Colon & 1.3 & 1.3 & 1.2 & 1.2 & 1.2 & 1.2 \\
$p$ & $.062^{*}$ & $.004 * *$ & .236 & $.034 * *$ & .157 & $.00 * *$ \\
\hline $\begin{array}{l}\text { Global (1+3) } \\
\text { Media }\end{array}$ & 2.0 & 2.5 & 1.8 & 1.9 & 2.6 & 2.7 \\
Media Colon & 1.01 & 1.1 & 1.3 & 1.3 & 1.3 & 1.3 \\
$p$ & $.016 * *$ & $.001 * *$ & $.046 * *$ & $.021^{* *}$ & $.060 *$ & $.00 * *$ \\
\hline p $<.1$ & & & & &
\end{tabular}

Diferencia entre las percepciones de riesgo de padecer diferentes tipos de cáncer entre los tratamientos 1 y 3. Prueba Z-Wilcoxon. 
Tabla 3

\begin{tabular}{llllll}
\hline & Total & Hombres & Mujeres & $\begin{array}{l}\text { Sí } \\
\text { anteceden }\end{array}$ & $\begin{array}{l}\text { No } \\
\text { anteceden }\end{array}$ \\
\hline $\begin{array}{l}\text { Rango promedio } \\
\text { tratamiento 2 }\end{array}$ & 15.7 & 4.6 & 11.1 & 7.8 & 8.4 \\
$\begin{array}{l}\text { Rango promedio } \\
\text { tratamiento } 4\end{array}$ & 14.3 & 5.2 & 9.8 & 8.8 & 5.1 \\
$p \quad .65$ & .90 & 0.66 & .86 & .15 \\
\hline $\begin{array}{l}\text { Diferencia entre las percepciones de riesgo de padecer cáncer de colon entre los tratamientos 2 y 4. } \\
\text { Prueba de U de Mann-Whitney. }\end{array}$
\end{tabular}


Tabla 4.

\begin{tabular}{llllll}
\hline Tratamiento 2 & Hombres & \multicolumn{3}{c}{ Mujeres } \\
& Pulmón & Próstata & Pulmón & Útero & Mama \\
\hline Media & 2.50 & 2.6 & 2.50 & 1.70 & 2.35 \\
Media Colon & 2.00 & 2.00 & 2.45 & 2.45 & 2.45 \\
$p$ & & .66 & .95 & .41 & .29 \\
\hline Tratamiento 4 & & & & & \\
Media & 2.5 & 2.25 & 3 & 1.77 & 2.6 \\
Media Colon & 2.16 & 2.16 & 2.22 & 2.22 & 2.22 \\
$p$ & & 1.00 & .10 & .32 & 1.00 \\
\hline Global (2+4) & & & & & \\
Media & 2.64 & 2.35 & 2.75 & 1.73 & 2.43 \\
Media Colon & 2.27 & 2.27 & 2.35 & 2.35 & 2.35 \\
$p$ & .16 & .79 & .13 & .21 & .36 \\
\hline
\end{tabular}

Diferencia entre las percepciones de riesgo de padecer diferentes tipos de cáncer entre los tratamientos 2 y 4 . Prueba Z-Wilcoxon. 
Tabla 5.

\begin{tabular}{lccccccc}
\hline & Reverte & Cano & Arias & Cruyff & Prats & Domingo & Posadas \\
\hline Agrado & .77 & .55 & .76 & .51 & .86 & .89 & .71 \\
Confianza & .75 & .51 & .68 & .48 & .81 & .82 & .68 \\
Credibilidad & .80 & .50 & .70 & .47 & .82 & .82 & .71 \\
\hline
\end{tabular}

Percepción de los personajes famosos 
Figura 2.

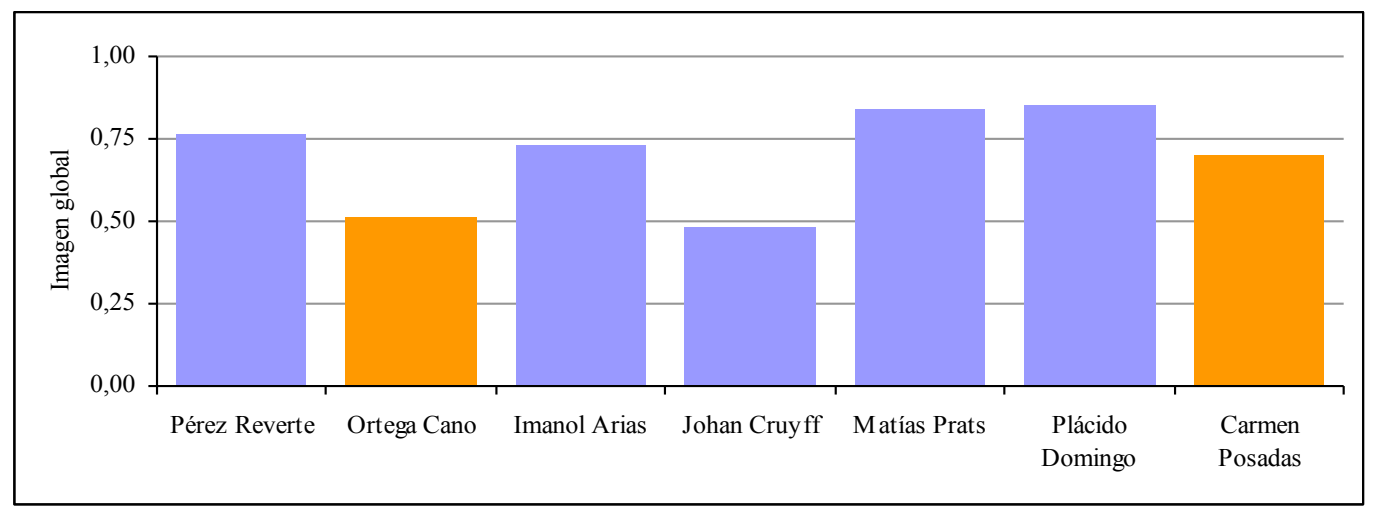

Imagen global de los personajes famosos 